\title{
Teorias e práticas de retorno e permanência no trabalho: elementos para a atuação dos terapeutas ocupacionais
}

\section{Theories and practices of return and permanence at work: elements for the performance of occupational therapists}

\author{
Selma Lancman ${ }^{1}$, Juliana de Oliveira Barros ${ }^{2}$, Tatiana de Andrade Jardim ${ }^{3}$
}

http://dx.doi.org/10.11606/issn.2238-6149.v27i2p101-108

\begin{abstract}
Lancman S, Barros JO, Jardim TA. Teorias e práticas de retorno e permanência no trabalho: elementos para a atuação dos terapeutas ocupacionais. Rev Ter Ocup Univ São Paulo. 2016 maio-ago.;27(2):101-8.
\end{abstract}

RESUMO: O trabalho é um dos aspectos determinantes dos processos saúde e doença, além de constituir-se como elemento central na construção da saúde e da identidade dos indivíduos. Dessa forma, tanto seus aspectos objetivos, como as condições de trabalho, tanto os subjetivos e relacionais, ligados à organização do trabalho constituem-se como pontos importantes de ancoragem para construção de programas de retorno ao trabalho que sejam realmente efetivos e aliem a necessidade da produção de bens e serviços à construção da saúde dos trabalhadores. A partir de um diálogo com a literatura nacional e internacional busca-se nesse artigo contribuir com o debate acerca dos aspectos relacionados ao retorno e a permanecia no trabalho e criar elementos de reflexão para as práticas e teorias da Terapia Ocupacional nesse campo de atuação.

DESCRITORES: Terapia ocupacional; Saúde e Trabalho; Retorno ao trabalho; Serviços de Saúde do trabalhador; Saúde do trabalhador; Saúde; Trabalho.
Lancman S, Barros JO, Jardim TA. Theories and practices of return and permanence at work: elements for the performance of occupational therapists. Rev Ter Ocup Univ São Paulo. 2016 May-Aug.;27(2):101-8.

ABSTRACT: The Work is one of the determining factors in the health-sickness process, and is also the central element in the construction of health and identity of individuals. Thus, both its objective aspects, such as working conditions, and the subjective and relational ones, related to the organization of work, are important for the construction of return-to-work programs that are really effective and combine the need for production of goods and services for the construction of the workers' health. Dialoguing with the national and international literature, the aim of this article is to contribute to the debate about the aspects related to return and permanence and to create elements of reflection to the practices and theories of the Occupational Therapy in this field.

KEYWORDS: Occupational therapy; Return to work; Occupational health services; Occupational health; Health; Work.

1. Profa.Titular do Departamento de Fisioterapia, Fonoaudiologia e Terapia Ocupacional da Faculdade de Medicina da USP. E-mail: lancman@usp.br

2. Terapeuta Ocupacional do Departamento de Fisioterapia, Fonoaudiologia e Terapia Ocupacional da Faculdade de Medicina da USP, doutora em Ciências da reabilitação pela FMUSP. E-mail: juliana.obarros@usp.br

3. Terapeuta Ocupacional do Departamento de Fisioterapia, Fonoaudiologia e Terapia Ocupacional da Faculdade de Medicina da USP, doutora em Ciências da reabilitação pela FMUSP. E-mail: tati.ajardim@usp.br

Endereço para correspondência: FOFITO. Curso de Terapia Ocupacional. R. Cipotânea, 51 - Vila Universitíaria, São Paulo, SP. CEP: 05360-000. 


\section{INTRODUÇÃO}

\} \begin{array} { l } { \text { o Brasil, a atuação da Terapia Ocupacional } } \\ { \text { no campo "saúde e trabalho" está } } \\ { \text { relacionada à própria criação da profissão } } \end{array} Desde então, ao longo dos anos, as práticas modificaramse em consonância com as transformações das políticas públicas que caminharam da medicina do trabalho, passando pela saúde ocupacional e, finalmente, à saúde do trabalhador ${ }^{1}$.

Vale destacar o caráter complexo da saúde do trabalhador que, necessariamente requer o engajamento de equipes multidisciplinares no desenvolvimento das ações sejam elas de prevenção, assistência, reabilitação ou retorno ao trabalho. Para a Terapia Ocupacional, tal multidisciplinariedade se dá simultaneamente pela aproximação com os saberes advindos de áreas distintas, tais como, saúde coletiva, ergonomia, psicologia social e do trabalho e psicodinâmica do trabalho, que passaram a integrar, progressivamente, os currículos de cursos de graduação e pós-graduação no Brasil².

Dessa forma, os terapeutas ocupacionais ampliaram seu campo de ação atuando, igualmente, na promoção da saúde do trabalhador e na prevenção de adoecimentos relacionados ao trabalho a partir de intervenções diretas nas situações laborais ${ }^{2}$. Esta aproximação acontece principalmente nos âmbitos da Vigilância em Saúde do Trabalhador e da Reabilitação profissional, com destaque aos aspectos do retorno e da permanência no trabalho enquanto etapas deste processo ${ }^{2,3}$.

Outro aspecto relevante é que, apesar da atuação da Terapia Ocupacional no campo da saúde do trabalhador propor ações especificas e desenvolver teorias e práticas singulares, a relação entre saúde e trabalho é transversal a toda e qualquer ação em terapia ocupacional e, portanto, vários dos aspectos abordados nessa área estão presentes e contribuem com o conjunto das práticas desenvolvidas em Terapia Ocupacional. Isto significa considerar a importância do trabalho e sua influência em todos os âmbitos do viver, ou seja, compreender sua centralidade na organização social e psíquica dos indivíduos, na determinação da qualidade de vida e do tempo do não trabalho ${ }^{4}$.

Desta forma, entendemos, que aprofundar a reflexão e o debate entre terapeutas ocupacionais a fim de que possamos aprimorar nossas praticas, quer seja em termos teóricos ou metodológicos é primordial.

Nesse artigo, destacamos especificamente os aspectos relacionados ao retorno e permanência no trabalho, por entendermos tratar-se de uma das etapas mais frágeis das políticas públicas em saúde do trabalhador ${ }^{5}$.
Ademais, retorno e permanência no trabalho são campo de atuação promissor para os terapeutas ocupacionais, tendo em vista: a crescente inserção dos terapeutas ocupacional no programa de reabilitação profissional do INSS em decorrência do número significativo de pessoas que se afastam do trabalho anualmente (Anuário estatístico do $\mathrm{INSS}^{6}$ ) e; da especificidade deste profissional que tem como objeto principal de estudo e a intervenção o fazer, aspecto fundamental na determinação dos processos saúde-doença ${ }^{5}$.

Para tanto, buscamos contribuições sobre o tema na literatura internacional e nacional que possam servir como base para o desenvolvimento de reflexões e das práticas dos Terapeutas Ocupacionais neste campo.

\section{CONTRIBUIÇÕES PARA O DEBATE NO CAMPO DO RETORNO E PERMANÊNCIA NO TRABALHO}

\section{A centralidade do trabalho}

O conceito de trabalho é complexo e seu sentido varia ao longo do tempo e de uma sociedade para outra. Além disso, a definição de trabalho é multidimensional e convoca diferentes disciplinas de tal forma que nenhuma delas tem o monopólio do objeto "trabalho"4,7,8.

As novas tecnologias, as transformações na organização e nos modos de produção, nas formas de vínculos e de pertencimento, de avaliação do trabalho, etc. vem promovendo profundas alterações no trabalhar e nas relações que dele decorrem. Essas mudanças influenciam diretamente o modo de trabalhar, os perfis de desgaste e adoecimento no trabalho, aumentando os afastamentos e dificultando os processos de retorno ao trabalho. Além disso, vem ocorrendo um crescimento das preocupações com os custos desses adoecimentos seja para o sistema previdenciário e produtivo, quanto para os próprios trabalhadores. O desenvolvimento sustentável, baseado em uma maior equidade social, econômica e ambiental, que inclui um trabalho saudável, digno e sustentável para aqueles que o realizam, passa a ganhar espaço no debate atual.

Muitos autores discutem a centralidade do trabalho no mundo social, sua importância nas relações indivíduosociedade e na constituição do próprio indivíduo e, apesar de tratarem essa centralidade de maneiras diversas, todos eles concordam com relação à importância do trabalho na constituição do indivíduo ${ }^{7,9,10,11}$.

O trabalho é mais do que o ato de trabalhar ou a busca de remuneração. Há também o reconhecimento 
social pelo trabalho, ou seja, o trabalho enquanto fator de pertinência a grupos, de participação e de acesso a certos direitos sociais.

O trabalho possui, ainda, uma função psíquica, enquanto um forte alicerce da constituição do sujeito e da sua rede de significados. Processos como reconhecimento, gratificação, mobilização da inteligência, além de estarem relacionados à realização do trabalho, estão ligados à constituição da identidade e da subjetividade ${ }^{4,7,8}$.

As mudanças atuais no trabalho e nas relações que dele decorrem provocam impacto na vida de indivíduos que são obrigados a conviver com lógicas de mercado e legislações que estão em constante transformação, criando uma situação de instabilidade e de ameaça que é vivenciada como um mal inevitável dos tempos modernos, atribuídas ao destino, à economia, ao mercado, ou ainda às relações sistêmicas. Da mesma forma, a precarização do trabalho, os processos de exclusão, a sobrecarga dos que permanecem tem gerado adoecimentos e retirado do trabalho uma parcela importante de trabalhadores em idade ativa.

Se o trabalho leva ao sofrimento e ao adoecimento, esse mesmo trabalho pode se constituir em uma fonte de prazer e de desenvolvimento psicossocial do indivíduo. Dessa forma, fica evidente que o trabalho e as relações que nele se originam nunca podem ser tomadas como um espaço de neutralidade subjetiva ou social e que o trabalho é também reconhecido como lócus privilegiado de integração social. Ele permite partilhar experiências com os outros e se implicar em atividades que ultrapassam o interesse individual, adquirindo status social e identidade profissional e social que envolvem o indivíduo em um número maior de redes sociais.

Como bem demonstrado pela ergonomia, diante da defasagem e da imprevisibilidade entre o trabalho prescrito (tarefa) e o trabalho real (atividade) existente em qualquer organização, as pessoas necessitam adaptar, criar e até mesmo desrespeitar certas orientações dadas pelos manuais, normas e pelos gestores sobre a maneira de trabalhar ${ }^{12}$.

Esse processo é essencial para que o trabalho possa acontecer. É necessário que as pessoas possam compartilhar, reinterpretar as orientações e a organização prescrita, reinventar coletivamente novas maneiras de trabalhar e, para que isso ocorra, a cooperação é essencial ${ }^{4,7.8}$. Isso requer que as equipes tenham tempo e espaço para discutir, deliberar, confrontar as diferentes formas de realizar o trabalho e como cada um reinterpreta as prescrições. Esse espaço permite a criação de acordos entre os diferentes membros de uma equipe ou de um coletivo para que construam uma interpretação comum das ordens e normas e um mesmo modo operatório. Isso é o que Dejours ${ }^{7}$ denomina de inteligência no plural. Para o autor, este espaço de deliberação é estruturado como espaço público, que faz com que o trabalho seja também importante espaço para o exercício da democracia. Para ele, "trabalhar é a atividade manifestada por homens e mulheres para realizar o que ainda não está prescrito pela organização do trabalho".

O trabalho é um elemento central na constituição da saúde, da identidade e o principal elo entre indivíduos e sociedade. Ou seja, compreender a importância do trabalho e seus efeitos sobre a psiquê significa dar visibilidade aos aspectos subjetivos mobilizados no ato de trabalhar. Trabalhar significa pensar, conviver, agir, construir-se a si próprio e confrontar-se perante o mundo. O sofrimento engendrado pelo trabalho é inerente a esse processo de confronto identitário e não será necessariamente patológico, ao contrário, dependendo das condições que o trabalhador tem de superá-lo poderá ser fator de crescimento e de desenvolvimento psíquico. Trabalhar pode promover o equilíbrio psíquico, a identificação com aquilo que se faz, a realização de si, porque é um meio essencial para a busca do sentido. Em suma, o trabalho é elemento central na construção da saúde. Isto reforça a ideia que o conceito de saúde deve ser entendido como processo, que perdura ao longo da vida e, que está relacionado com as possibilidades que o sujeito tem para agir no mundo, isto é, as possibilidades que cada um tem para construir, no seu meio social e nos coletivos onde vive, a sua vida ${ }^{4,7,8}$.

Nesse sentido, a inclusão, os processos de afastamento e exclusão do trabalho e a necessidade de práticas que assegurem o retorno e a permanência no trabalho tornam-se fundamentais para os indivíduos enquanto fator de pertencimento e participação social e de desenvolvimento psíquico.

\section{Processos de adoecimento, afastamento e retorno ao trabalho}

A constatação dos efeitos deletérios do trabalho na saúde e suas consequências no aumento dos adoecimentos, nos crescentes afastamentos e nas dificuldades de retorno e permanência no trabalho revelam a importância de reflexões e fomento de políticas públicas. É necessário compreender os aspectos relativos à promoção e prevenção da saúde, os adoecimentos relacionados ao trabalho e às possibilidades de tratamento, reabilitação, retorno e permanência, enquanto aspectos indissociáveis s $^{3,4,5,13,14}$. 
No Brasil, o aumento do número de adoecimentos e de afastamentos relacionados ao trabalho têm crescido nos últimos anos, devido aos novos processos de organização e das condições de trabalho Muitos desses processos estão associados à precarização do trabalho, redução do número de trabalhadores e novas normas de avaliação do trabalho, em especial a avaliação individual de performance ${ }^{15}$.

Em geral, a maior parte dos afastamentos ocorre por questões físicas (problemas osteomusculares), transtornos psíquicos e acidentes do trabalho ${ }^{6}$.

Elevados números de afastamentos são um sinal de alerta para a identificação de situações de trabalho adoecedoras e para a necessidade de mudanças visando a prevenção de novos adoecimentos e a sustentação do retorno ao trabalho como processo efetivo e duradouro ${ }^{16}$.

A dinâmica dos adoecimentos, afastamentos e retornos ao trabalho tem relação direta com o próprio trabalho e com as relações que dele decorrem. Mesmo quando o trabalho não é o principal fator de adoecimento, e consequentemente de afastamento, sua organização e seus atores são decisivos nos processos de retorno e permanência ${ }^{5}$.

Sendo assim, ressalta-se que diversos fatores influenciam e dificultam os processos de retorno e permanência no trabalho. Entre os mais conhecidos estão àqueles relacionados aos agravos e restrições de saúde, ao tempo de duração da ausência e a ocorrência de sucessivos afastamentos. Porém, sobressaem-se os aspectos do trabalho que ocasionaram ou que agravaram o problema de saúde dos trabalhadores e que não são transformados para acolher aqueles em processo de retorno, favorecendo, portanto, dificuldades de permanência e novas situações de afastamento ${ }^{17}$.

Destacam-se, neste contexto, tanto os aspectos objetivos do trabalho, tais como as condições materiais, quanto os organizacionais, tais como: a divisão de tarefas e das pessoas num determinado processo de produção, a estrutura hierárquica, os tempos de trabalho e pausa, os ritmos, exigências de qualidade e de produtividade. Ressaltam-se ainda os aspectos subjetivos e relacionais, ou seja, processos de interação entre pares e entre pares e chefias ${ }^{12}$.

O funcionamento global das situações de trabalho e a complexidade dos aspectos subjetivos e relacionais decorrentes da necessidade de integração entre os atores têm sido desconsideradas nos processos de retorno, gerando soluções e ações parciais e improvisadas para dar conta do cotidiano das atividades. Tais processos, aliados à falta de programa de apoio ao retorno e permanência no trabalho, culminam em novos afastamentos, na não reintegração dos trabalhadores que retornam, em demissões e aposentadorias precoces, transformando processos de retorno ao trabalho em processos de exclusão $0^{5,18,19,20}$.

Nesse sentido, o afastamento, o retorno e a permanência no trabalho, precisam transcender uma perspectiva numérica, ou seja, de que se trata apenas de uma divisão entre quantidade de trabalho e número de trabalhadores disponíveis nos contextos profissionais.

Para construção de programas de retorno e permanência no trabalho que sejam realmente efetivos e aliem a necessidade da produção de bens e serviços à construção da saúde dos trabalhadores, é necessário uma compreensão mais ampla desses elementos. Tal compreensão engloba: os efeitos patogênicos do trabalho nas pessoas, e o adoecimento de um determinado trabalhador como sinal de que uma determinada situação trabalho pode afetar a saúde de outras pessoas, as possíveis interferências do afastamento de um par para toda a equipe e de como o trabalhador que retorna, mais do que voltar a exercer determinadas tarefas, terá que (re) compor um coletivo de trabalho, entre outros ${ }^{5,21}$.

\section{Processos de retorno e permanência no trabalho: apontamentos da literatura}

Na perspectiva da (re) integração dos trabalhadores no processo de retorno e, consequentemente, da permanência no trabalho, considera-se fundamental a sistematização de propostas de ação efetivas que possam favorecer este processo. Estudos nacionais e internacionais têm problematizado alguns aspectos a serem considerados.

No momento do retorno ao trabalho, os trabalhadores devem retornar á função de origem ou em função compatível com sua condição de saúde e capacidade laborativa. O sucesso ou fracasso desse processo dependerá de diversos aspectos, entre eles: condições e organização do trabalho, relações interpessoais (como por exemplo, a resistência dos colegas em receber o trabalhador que retorna), capacidade laborativa (avaliação da capacidade laborativa naquele determinado momento e da existência ou não de restrição laboral), capacitação profissional (falta de capacitação e formação para o desenvolvimento de outra função), rápida obsolescência de postos de trabalho (por transformações tecnológicas ou mudanças nas exigências do mercado). Todos estes aspectos são necessários para a realização do trabalho e interferem de forma positiva ou negativa na possibilidade de acolhimento daqueles que retornam $^{5,22,23,24}$.

Dependerá, ainda, da forma como o retorno é conduzido pela instituição, com a participação ou não dos 
serviços de saúde e do próprio trabalhador ${ }^{21,25,26}$. Esses aspectos evidenciam que o êxito de ações de retorno e permanência no trabalho estão relacionados com o envolvimento de diversos órgãos, campos, profissionais e atores sociais, que devem direcionar suas atuações em um eixo comum e coletivamente pré-definido ${ }^{5,21}$.

Estudos apontam que, quanto maior a necessidade de interação social nos ambientes de trabalho maior a importância da equipe para acolher os trabalhadores que retornam. Ademais, o tempo de afastamento fragiliza as relações entre pares e os processos de cooperação dificultando ainda mais o retorno e a permanência no trabalho ${ }^{27,28}$.

Os afastamentos, tanto de curta quanto de longa duração e os trabalhadores que retornam com restrições, ou seja, com sua capacidade de trabalho limitada para o desenvolvimento de determinadas atividades, fragilizam o planejamento do trabalho que precisa ser reorganizado para viabilizar o desenvolvimento do mesmo volume de atividades. No entanto, mais do que uma divisão de trabalho mais ou menos equitativa, o ponto central é a cooperação, as possiblidades das equipes poderem compreender os processos de adoecimento e retorno e, desta forma, transformá-los em processos de promoção da saúde e prevenção de adoecimento de todos. A criação de estratégias que estimulem a percepção de justiça distributiva, isto é, de que há uma equidade na distribuição das tarefas entre o conjunto de trabalhadores é fundamental. Os pares podem aceitar uma distribuição diferenciada (criada pela necessidade de acomodação de um trabalhador com restrição) se as restrições forem percebidas como necessárias ou justas ${ }^{17}$.

As empresas não garantem a reposição das pessoas afastadas ou com restrições laborais, que são contabilizadas no dimensionamento da equipe. Esta situação, aliada às metas de produção, traz impactos para os demais trabalhadores, que assumem maior número de tarefas, normalmente aquelas que provocam maior sobrecarga, dificultando o processo de reinserção laboral, a construção de laços de solidariedade e de cooperação em relação aos que retornam ${ }^{21,29,30}$.

Durand et al. ${ }^{31}$ alertam para a falta de margem de manobra dos contextos institucionais, para a padronização das formas de trabalho e para a falta de autonomia que o trabalhador tem de desenvolve-lo de diferentes formas a fim de atingir metas de produção. Em geral, os processos de trabalho são determinados por dois fatores: as características do trabalhador (por exemplo, a sua capacidade para o trabalho) e as exigências do trabalho e os meios e condições oferecido para o desempenho da tarefa. Embora o conceito de margem de manobra seja amplamente estudado em ergonomia ele não tem sido utilizado na reintegração de trabalhadores com restrição laboral, na reorganização das equipes e na sustentabilidade do trabalho ${ }^{12}$

O planejamento do retorno ao trabalho deve ser reconhecido como um processo social frágil e, deve garantir que não haja prejuízos e sobrecarga dos pares. $\mathrm{O}$ necessário apoio oferecido por eles está relacionado ao sentimento de justiça quanto à divisão do trabalho $\mathrm{e}$ dos recursos instrumentais. No entanto, este julgamento é relativizado a depender do grau de empatia que possuem com o trabalhador com restrições e da compreensão das relações de trabalho com os processos de adoecimento ${ }^{17,32}$.

Dessa forma, as relações de cooperação e a solidariedade das equipes são fundamentais para os processos de retorno e permanência. Considera-se, nesse contexto, que o retorno ao trabalho e, sobretudo, a permanência nele, como um processo que pode favorecer as relações de toda a equipe, pois há a possibilidade de repensar a situação de trabalho e, consequentemente, as transformações ali possíveis, de forma global. Tornase evidente ainda a necessidade de tornar visível a influência dos pares neste processo, fundamentais para a sustentabilidade do trabalhador na situação de trabalho ${ }^{22,23}$.

A inter-relação entre aspectos organizacionais, relacionais e o retorno ao trabalho, compõe um processo dinâmico e interativo no qual retornar ao trabalho gera transformações nos âmbitos organizacional e relacional e estes impactam diretamente nas possibilidades de retorno e permanência. Além disso, evidencia-se que a ausência de profissionais especializados que gerenciem esses processos e de programas e modelos teórico-metodológicos de retorno ao trabalho que se apliquem a realidade brasileira se configuram como um dos fatores que dificultam e até mesmo inviabilizem esses processos no cenário nacional.

Diversos estudos têm propostos modelos e propostas para auxiliar a reintegração no trabalho de trabalhadores que passaram por afastamentos enfatizando a importância dos pares na acomodação dos colegas que retornam ${ }^{17,22,23,24}$. Entre os aspectos descritos nesses modelos e programas de retorno, as ações devem ser precoces, pois quanto mais cedo forem implantadas, mais chances haverá de sucesso.

Além disso, as ações devem ser voltadas tanto para aspectos organizacionais quanto relacionais, devem levar à mudança de alguns paradigmas voltados, por exemplo, para as capacidades dos trabalhadores que retornam em detrimento das restrições e para a confluência entre ações de reabilitação profissional com a prevenção de novos acometimentos. 
Em suma, para que os processos de retorno e permanência no trabalho sejam bem-sucedidos, todos os elementos que compõem a situação laboral devem ser considerados como fatores fundamentais. Ou seja, chefias, pares, o trabalho (seu conteúdo, organização e condições) além do trabalhador e da própria estrutura organizacional da empresa. As mudanças na organização do trabalho (incluindo possíveis ajustes ergonômicos) e as relações sociais positivas entre os pares são um elemento determinante do sucesso desses processos ${ }^{17}$.

Compreender o trabalho real, as condições e organização do trabalho, assim como os aspectos relacionais da equipe de trabalho e dos diferentes níveis hierárquicos são elementos essenciais e indissociáveis para garantir a qualidade do serviço prestado e, ao mesmo tempo, a saúde dos trabalhadores, incluindo os processos de retorno e permanência no trabalho ${ }^{17}$.

Em relação às possíveis limitações laborais dos que retornam, deve-se levar em conta que a experiência e o tempo de trabalho desenvolvem a inteligência e que as competências aumentam ao longo dos anos. Dessa forma, o envelhecimento no trabalho, a perda de habilidades, podem ser compensadas com o desenvolvimento de capacidades mais refinadas, da experiência, do saberfazer. Como o trabalho não se dá somente por uma relação individual com a tarefa, mas a partir de uma relação que também é coletiva, os mais velhos e os trabalhadores com eventuais restrições laborais podem tornar-se úteis pela sua função na equipe ${ }^{7}$.

Se a empresa se organiza a partir de metas rígidas estabelecidas por hora, ou dias, ou com quadros enxutos onde a impossibilidade de um trabalhador desenvolver certa atividade não permite margens de manobra para que a equipe reorganize seu trabalho, não priorize grupos que trabalhem de forma cooperativa, a atuação dos envolvidos com a reabilitação profissional e com o retorno ao trabalho terá sua ação muito reduzida.

Os processos de acompanhamentos do retorno ao trabalho, apesar de individuais, pressupõem mudanças organizacionais. Implicam no envolvimento de diretores de empresas, departamentos de recursos humanos, de saúde e segurança no trabalho com a saúde dos trabalhadores e sua permanência no trabalho. Dessa forma os programas envolvem, além de intervenções individuais, um processo integrado de saúde no trabalho que considere a complexidade do ser humano e a necessidade de agir não somente junto ao trabalhador mas igualmente no coletivo, nas condições e na organização do trabalho. È necessário para a implantação do programa, mudar a cultura das empresas de seus valores e metas relativas ao apoio a pessoas e às práticas de gestão de afastamentos e retorno ao trabalho ${ }^{21}$.

\section{CONSIDERAÇÕES FINAIS: AÇÃO DA TERAPIA OCUPACIONAL NO CAMPO DO RETORNO E PERMANÊNCIA NO TRABALHO}

O retorno e, sobretudo, a permanência no trabalho daqueles que adoeceram ou sofreram acidentes do trabalho deveria ser a ponta final do processo de reabilitação profissional ${ }^{3}$, mas, na prática, se configura como uma das questões mais desconsideradas. Soma-se a isso a dificuldade em garantir os direitos do trabalhador para que, de volta ao trabalho, ele não seja demitido ou "encostado" e que, desta forma, o retorno se transforme em um processo de exclusão tardia.

O retorno e a permanência no trabalho só ocorrerão se levarmos em consideração os fatores que causaram o adoecimento e a necessidade de transformá-los. São diferentes etapas de um mesmo processo que é contínuo e único: cuidar do retorno ao trabalho, conhecer onde o processo de adoecimento começou e intervir no trabalho para prevenir novos adoecimentos. Significa também agir na empresa, nos departamentos de recursos humanos e de saúde do trabalhador para que se crie uma cultura e uma sinergia que transforme os programas de retorno em prevenção à saúde dos trabalhadores.

A dificuldade de inserir no mundo do trabalho pessoas que por diversos motivos estão afastadas desse espaço social é uma antiga preocupação dos Terapeutas Ocupacionais, que começaram, mais recentemente, a compor equipes nos Departamentos de Saúde Ocupacional e nos Serviços Especializados em Engenharia de Segurança e Medicina do Trabalho (SESMT) das empresas e vêm colaborando na prevenção de agravos à saúde do trabalhador e na promoção da saúde no e pelo trabalho.

Trata-se de intervenções que previnem afastamentos ou aposentadorias precoces e auxiliam na percepção de risco de acidentes do trabalho ou adoecimentos, a partir, por exemplo, do desenvolvimento de programas de retorno ao trabalho, com vistas à realocação de indivíduos com restrições ocupacionais, decorrentes de processos de desgaste ou adoecimento. São desenvolvidas com base nos conceitos trazidos pela Classificação Internacional de Funcionalidade, Incapacidade e Saúde (CIF) para, a partir de uma avaliação complexa e singular, contribuir na compatibilização e diálogo entre as condições de saúde das pessoas e as exigências das atividades de trabalho.

Ademais, a literatura internacional propõe que, ainda na fase do afastamento e da recuperação clínica, 
Lacman S, et al. Teorias e práticas de retorno e permanência no trabalho. Rev Ter Ocup Univ São Paulo. 2016 maio/ago.;27(2):101-8.

exista um gestor que acompanhe o trabalhador desde o afastamento até o processo de retorno e permanência no trabalho, realizando uma interlocução junto aos diversos atores envolvidos no processo (diferentes níveis hierárquicos da empresa, pares, chefias, INSS, etc). Entendemos que estas ações configuram um campo ainda pouco explorado no contexto nacional, porém, destacamos que os Terapeutas Ocupacionais são profissionais com um perfil impar para ocupar este espaço no Brasil.

\section{REFERÊNCIAS}

1. Daldon MTB, Lancman S. Vigilância em saúde do trabalhador: rumos e incertezas. Rev Bras Saude Ocup. 2013;38(127):92-106. DOI: http://dx.doi.org/10.1590/ S0303-76572013000100012.

2. Lancman S, Ghirardi MIG. Pensando novas praticas em terapia ocupacional, saúde e trabalho. Rev Ter Ocup Univ São Paulo. 2002;13(2):44-85. DOI: http://dx.doi.org/10.11606/ issn.2238-6149.v13i2p44-50.

3. Maeno M, Takahashi MAC, Lima MAG. Reabilitação profissional como política de inclusão social. Acta Fisiatr. 2009;16(2): 53-8. http://www.actafisiatrica.org.br/detalhe artigo.asp? $\mathrm{id}=108$.

4. Lancman S, Sznelwar LI. Christophe Dejours: da psicopatologia à psicodinâmica do trabalho. Brasília: Paralelo15; 2004.

5. Lancman S, Daldon MT, Barros J, Gonçalves R, Jardim T. Processos de retorno e permanência no trabalho: elementos estruturantes para a construção de um modelo de ação. In: Simonelli AP, Rodrigues DS. Saúde e trabalho em debate: velhas questões, novas perspectivas. Brasília: Paralelo 15; 2013. p.135-58.

6. Brasil. Ministério da Previdência Social. Anuário estatístico da Previdência Social. Brasília; 2013.

7. Dejours C.Trabalho e emancipação: trabalho vivo tomo II. Brasilia: Paralelo 15, 2012.

8. Lancman S, Uchida S, Sznelwar L. Contribuições da psicodinâmica do trabalho para o desenvolvimento de ações transformadoras. In: Glina DMR, Rocha LE. Saúde mental no trabalho, da teoria à prática. São Paulo: Roca; 2010. p.191-209.

9. Antunes R. Adeus ao trabalho? Ensaio sobre as metamorfoses e a centralidade do mundo do trabalho. 6a ed. São Paulo: Cortez; 1999
Estamos diante de um campo complexo e inovador. Acreditamos que ainda há muito a ser desenvolvido tanto do ponto de vista teórico quanto metodológico. Considerar a complexidade dos aspectos apontados nas intervenções da terapia ocupacional em saúde do trabalhador ainda é um desafio para nós, terapeutas ocupacionais. No entanto, acreditamos que é a partir da nossa atuação nesta área e da reflexão disparada a partir dela que podemos avançar.

10. Castel R. As metamorfoses da questão social: uma crônica do salário. Trad. Iraci D. Poleti. Petrópolis: Vozes; 1998.

11. Sennett R. A corrosão do caráter: consequências pessoais do trabalho no novo capitalismo. Rio de Janeiro: Record; 1999.

12. Abrahão J, Sznelwar L, Silvino A, Sarmet M, Pinho D. Introdução à Ergonomia: da prática à teoria. São Paulo: Blucher; 2009.

13. Dias, EC. Organização da Atenção à Saúde no Trabalho. In: Ferreira, JM. Saúde no trabalho: temas básicos para o profissional que cuida dos trabalhadores. São Paulo: Roca; 2002. p.3-28.

14. Minayo-Gomes C, Thedim-Costa SM. A construção do campo da Saúde do Trabalhador: percurso e dilemas. Cad Saúde Pública. 1997;13(2):21-32. DOI: http://dx.doi. org/10.1590/S0102-311X1997000600003.

15. Dejours, C, Avaliação do trabalho submetida à prova do real: criticas aos fundamentos da avaliação. São Paulo: Ed Blucher; 2008.

16. Van Oostrom SH, Driessen MT, De Vet HC, Franche RL, Schonstein E, Loisel $\mathrm{P}$, et al. Workplace interventions for preventing work disability. Cochrane Database Syst Rev. 2009;15(2): CD006955. DOI: 10.1002/14651858. CD006955.pub2.

17. Lancman S, Barros JO, Silva MD, Pereira AR, Jardim TA. Interrelationship between organizational and relational aspects and the return-to-work process: a case study with nursing professionals at a teaching hospital in Brazil. J Occup Rehabil. 2016;1-10. DOI: 10.1007/s10926-016-9631-8.

18. Saint-Arnaud L, Saint-Jean M, Damasse J. Towards an enhanced understanding of factors involved in the returnto-work process of employees absent due to mental health problems. Can J Commun Mental Health. 2006;25(2):30315. Disponível em: http://www.integration-travail.fse.ulaval. ca/fichiers/site_chaire_ipept/documents/Texte_Louise/ Toward_enhanced_understanding_RTW_process.pdf. 
19. Saint-Arnaud L, Bourbonnais R, Saint-Jean M, Rhéaume, J. Determinants of return-to-work among employees absent due to mental health problems. Industrial Rel Q Rev. 2007;62(4):690-713. DOI: 10.7202/016957ar.

20. Saint-Arnaud L, Briand C, Durand M, Corbière M, Pelletier $\mathrm{M}$, Kedl E. The best practices for managing return to work following mental health problems at work. In: Clarke S, Burke R, Cooper C, editors. Inglaterra: Gower Publishing; 2011. p.343-57.

21. Saint-Arnaud L, Pelletier M. Guide soutenir le retour au travail et favoriser le maintien en employ. Institut de Recherche Robert-Sauvé en Santé et en Sécurité du Travail; 2012. http://www.irsst.qc.ca/media/documents/PubIRSST/ RG-758.

22. Tjulin A, MacEachen E, Ekberg K. Exploring Workplace actors experiences of the social organization of return-towork. J Occup Rehabil. 2010;20(3):311-21. DOI: 10.1007/ s10926-009-9209-9.

23. Tjulin Å, MacEachen E, Stiwne EE, Ekberg K. The social interaction of return to work explored from co-workers experiences. Disabil Rehabil. 2011;33(21-22):1979-89. DOI: $10.3109 / 09638288.2011 .5537082$.

24. Dunstan DA, MacEachen E. Bearing the brunt: co-workers' experiences of work reintegration processes. J Occup Rehabil. 2013;23(1):44-54. DOI: 10.1007/s10926-012-93802. doi: $10.1007 / \mathrm{s} 10926-012-9380-2$.

25. Loisel P, Durand P, Abenhaim L, Gosselin L, Simard R, Turcotte J, et al. Management of occupational back pain: the Sherbrooke model. Results of a pilot and feasibility study. J Occup Environ Med. 1994;51(9):597-602. Disponível em: http://www.ncbi.nlm.nih.gov/pmc/articles/PMC1128053/.

26. Arnetz BB, Sjögren B, Rydéhn B, Meisel R. Early workplace intervention for employees with musculoskeletal-related absenteeism: a prospective controlled intervention. J Occup Environ Med. 2003;45(5):499-506. DOI: 10.1097/01. jom.0000063628.37065.45.

27. Murphy GC, Foreman P. General patterns of managerial approaches to work motivation: implications for rehabilitation professionals involved in occupational rehabilitation. J Occup Rehabil. 1993;3(1):51-62. DOI: 10.1007/BF01076742.

28. Chiaburu DS, Harrison DA. Do Peers Make the Place? Conceptual synthesis and meta-analysis of coworker effects on perceptions, attitudes, OCBs, and performance. J Appl Psychol. 2008;93(5):1082-103. DOI: 10.1037/00219010.93.5.1082.

29. Briand C, Durand MJ, St-Arnaud L, Corbière M. Work and Mental Health: Learning from Return-to-Work Rehabilitation Programs Designed for Workers with Musculoskeletal disordres. Int J Law Psychiatry. 2007;30(4-5):444-57. DOI: 10.1016/j.ijlp.2007.06.014.

30. Toulouse G, Saint-Arnaud L, Bourbonnais R, Damasse J, Chicoine D, Delisle A. Étude de la prévalence des facteurs physiques et psychosociaux des troubles musculosquelettiques et psychologiques chez les préposés des centres d'urgence 9-1-1. França: Institut Robert Sauvé de Recherche sur la Santé et la Sécurité; 2007.

31. Durand MJ, Vézina N, Baril R, Loisel P, Richard MC, Ngomo S. Margin of manoeuvre indicators in the workplace during the rehabilitation process: a qualitative analysis. J Occup Rehabil. 2009;19(2):194-202. DOI: 10.1007/s10926009-9173-4.

32. Stone DL, Colella A. A Model of factors affecting the treatment of disabled individuals in organizations. Acad Manage Rev. 1996;21(2):352-401. DOI: 10.5465/ AMR.1996.9605060216.

Recebido: 30.10.15

Aceito: 08.02.16 